\title{
OH-chemistry of non-methane organic gases (NMOG) emitted from laboratory and ambient biomass burning smoke: evaluating the influence of furans and oxygenated aromatics on ozone and secondary NMOG formation.
}

Matthew M. Coggon ${ }^{1,2}$, Christopher Y. Lim ${ }^{3}$, Abigail R. Koss ${ }^{1,2, \ddagger}$, Kanako Sekimoto $^{1,2,4}$, Bin Yuan ${ }^{1,2, \dagger}$, Jessica B Gilman ${ }^{2}$, David H. Hagan ${ }^{3}$, Vanessa Selimovic ${ }^{5}$, Kyle Zarzana ${ }^{1,2}$, Steven Brown ${ }^{2}$, James M. Roberts ${ }^{2}$, Markus Müller ${ }^{6}$, Robert Yokelson ${ }^{5}$, Armin Wisthaler ${ }^{7,8}$, Jordan Krechmer ${ }^{9}$, Jose Jimenez $^{1,10}$, Christopher Cappa ${ }^{11}$, Jesse Kroll ${ }^{3}$, Joost de Gouw ${ }^{1,10}$, and Carsten Warneke ${ }^{1,2}$

${ }^{1}$ Cooperative Institute for Research in Environmental Sciences, University of Colorado, Boulder, CO, USA

${ }^{2}$ NOAA Earth Systems Research Laboratory Chemical Sciences Division, Boulder, CO, USA

${ }^{3}$ Department of Civil and Environmental Engineering, Massachusetts Institute of Technology, Cambridge, MA, USA

${ }^{4}$ Graduate School of Nanobioscience, Yokohama City University, Yokohama, Kanagawa, Japan

${ }^{5}$ Department of Chemistry and Biochemistry, University of Montana, Missoula, MT, USA

${ }^{6}$ Ionicon Analytik, Innsbruck, Austria

${ }^{7}$ Institute for Ion Physics and Applied Physics, University of Innsbruck, Innsbruck, Austria

${ }^{8}$ Department of Chemistry, University of Oslo, Oslo, Norway

${ }^{9}$ Aerodyne Research, Inc., Billerica, MA, USA

${ }^{10}$ Department of Chemistry, University of Colorado, Boulder, CO, USA

${ }^{11}$ Department of Civil and Environmental Engineering, University of California, Davis, CA, USA

${ }^{\ddagger}$ now Department of Civil and Environmental Engineering, Massachusetts Institute of Technology, Cambridge, MA, USA

${ }^{\dagger}$ now at Institute for Environment and Climate Research, Jinan University, Guangzhou, China.

Correspondence: Matthew Coggon (matthew.m.coggon@noaa.gov), Carsten Warneke (carsten.warneke@noaa.gov)

\section{Evaluating NOMOG Wall Losses Through Tubing and Gas-Wall Partitioning}

Prior to each small chamber experiment, the PTR-ToF-MS sampled NMOG directly from the stack, as described by Koss et al. (2018). To evaluate biases associated with NMOG transmission through the ductwork, we compare the distribution of NMOG measured from the stack with that inside the small chamber prior to the initiation of $\mathrm{OH}$ chemistry. Figure $\mathrm{S} 15$ shows the difference in NMOG profiles between small chamber and stack measurements (Small Chamber Bias $=\left[\mathrm{NMOG}_{\mathrm{i}} / \mathrm{CH}_{3} \mathrm{CN}\right]_{\text {chamber }}$ $\left[\mathrm{NMOG}_{\mathrm{i}} / \mathrm{CH}_{3} \mathrm{CN}\right]_{\text {stack }}$ ). We normalize the NMOG distribution to $\mathrm{CH}_{3} \mathrm{CN}$ under the assumption the acetonitrile is not lost to surfaces. The top row shows bias histograms for three fires, while the bottom row shows a comparison between the normalized profiles with 1:1, 1:2, and 2:1 lines.

In general, most NMOG fall within $20 \%$ of the $1: 1$ line. Bias histograms (top row, Fig. S15) show that the NMOG/CH $3 \mathrm{CN}^{2}$ ratios are lower in the small chamber, which suggests that NMOG are lost to the ductwork; however, this loss appears to be normally distributed and not weighted towards any specific NMOG functionality. Some masses exhibit significantly higher 
ratios with acetonitrile inside the small chamber (e.g. butenes, ethanol, formamide for F29); however, PTR-ToF-MS detection of these masses is poor due to contributions of fragments from higher masses (e.g. butene), or low sensitivity (e.g. ethanol).

Fig. S15 demonstrates that the NMOG distribution in the chamber is similar to the NMOG distribution sampled from the

15 stack. These results are consistent with the conclusions drawn by Lim et al. (2019), which showed that the volatility distribution was not significantly different between stack and small chamber measurements.

\section{Sensitivity of Modeled Secondary NMOG Formation to Furfural Branching Ratios}

The reactions employed to represent furfural oxidation were estimated by Zhao and Wang (2017) via theoretical quantum chemistry calculations. To date, this mechanism has not been studied experimentally; consequently, the exact branching ratios of the three major pathways may differ from those used in this study ( 0.37 for channel $\mathrm{A}, 0.6$ for channel $\mathrm{B}$, and 0.03 for channel $\mathrm{C}, 6$ ). Furfural plays a major role in the formation of secondary NMOG measured in the biomass burning plume described by Müller et al. (2016) and the assumed branching ratios may impact modeled formation of maleic anhydride, hydroxy furanone, and ozone.

Figure S16 shows model output of maleic anhydride, hydroxy furanone, and ozone for base case $(\mathrm{A}=0.37, \mathrm{~B}=0.6, \mathrm{~B}=$ $0.03)$, equal weight $(0.33,0.33,0.33)$, and isolated channel (i.e., all channel $\mathrm{A}, \mathrm{B}$, or $\mathrm{C}$ ) simulations of the biomass burning plume described by Müller et al. (2016). Overall, hydroxy furanone formation is most sensitive to the assumed branching ratio of channel $\mathrm{B}$, which is the pathway that directly leads to hydroxy furanone formation (Fig. 6). Maleic anhydride is most sensitive to the assumed branching ratio of channel C; however, this sensitivity is weaker than that of hydroxy furanone since all pathways lead to a significant yield of maleic anhyride. The assumed branching ratios have little impact on ozone formation.

The sensitivity tests presented in Fig. S16 demonstrate the need for experimental evaluation of the furfural oxidation mechanism. This refinement may provide better constraints of important secondary NMOG; however, this will unlikely affect modeled ozone formation. 


\section{References}

Aschmann, S. M., Nishino, N., Arey, J., and Atkinson, R.: Products of the OH Radical-Initiated Reactions of Furan, 2- and 3-Methylfuran, and 2,3- and 2,5-Dimethylfuran in the Presence of NO, J. Phys. Chem. A., 118, 457-466, 2014.

Koss, A. R., Sekimoto, K., Gilman, J. B., Selimovic, V., Coggon, M. M., Zarzana, K. J., Yuan, B., Lerner, B. M., Brown, S. S., Jimenez, J. L., Krechmer, J., Roberts, J. M., Warneke, C., Yokelson, R. J., and de Gouw, J.: Non-methane organic gas emissions from biomass burning: identification, quantification, and emission factors from PTR-ToF during the FIREX 2016 laboratory experiment, Atmospheric Chemistry and Physics, 18, 3299-3319, https://doi.org/10.5194/acp-18-3299-2018, 2018.

40 Lauraguais, A., Coeur-Tourneur, C., Cassez, A., Deboudt, K., Fourmentin, M., and Choël, M.: Atmospheric reactivity of hydroxyl radicals with guaiacol (2-methoxyphenol), a biomass burning emitted compound: Secondary organic aerosol formation and gas-phase oxidation products, Atmospheric Environment, 86, 155-163, 2014.

Lim, C., Hagan, D., Cappa, C., Coggon, M. M., Koss, A. R., Sekimoto, K., de Gouw, J., Warneke, C., and Kroll, J.: Secondary organic aerosol formation from biomass burning emissions, in prep, 2019.

45 Müller, M., Anderson, B. E., Beyersdorf, A. J., Crawford, J. H., Diskin, G. S., Eichler, P., Fried, A., Keutsch, F. N., Mikoviny, T., Thornhill, K. L., Walega, J. G., Weinheimer, A. J., Yang, M., Yokelson, R. J., and Wisthaler, A.: In situ measurements and modeling of reactive trace gases in a small biomass burning plume, Atmospheric Chemistry and Physics, 16, 3813-3824, 2016.

Sekimoto, K., Li, S.-M., Yuan, B., Koss, A., Coggon, M., Warneke, C., and de Gouw, J.: Calculation of the sensitivity of proton-transferreaction mass spectrometry (PTR-MS) for organic trace gases using molecular properties, International Journal of Mass Spectrometry, 421, 71-94, 2017.

Zhao, X. and Wang, L.: Atmospheric Oxidation Mechanism of Furfural Initiated by Hydroxyl Radicals, J. Phys. Chem. A., 121, 3247-3253, 2017. 
Table S1. Rate constant parameters, photolysis frequency, and initial conditions for the species modeled in F26 and F38. Entries are ordered by mixing ratios measured during F38. Photolysis frequencies are calculated based on literature cross-sections, known/estimated quantum yields, and the scaled photon flux at $254 \mathrm{~nm}\left(4.5 \times 10^{15}\right.$ photons $\left.\mathrm{cm}^{2}\right)$.

\begin{tabular}{|c|c|c|c|c|c|c|c|}
\hline & MCM Name & $\mathrm{k}_{\mathrm{OH}}\left(\mathrm{cm}^{3} \mathrm{molec}^{-1} \mathrm{~s}^{-1}\right)$ & $\mathrm{k}_{\mathrm{O} 3}\left(\mathrm{~cm}^{3} \mathrm{molec}^{-1} \mathrm{~s}^{-1}\right)$ & $\mathrm{k}_{\mathrm{NO} 3}\left(\mathrm{~cm}^{3} \mathrm{molec}^{-1} \mathrm{~s}^{-1}\right)$ & $\mathrm{j}\left(\mathrm{s}^{-1}\right)$ & F26 (ppb) & F38 (ppb) \\
\hline d-butanol & DBUTANOL & $3.40 \mathrm{E}-12$ & 0 & 0 & 0 & 37.26 & 44.86 \\
\hline Formaldehyde & $\mathrm{HCHO}$ & $8.47 \mathrm{E}-12$ & 0 & $5.50 \mathrm{E}-16$ & $1.20 \mathrm{E}-05$ & 14.07 & 17.84 \\
\hline 1-butene & BUT1ENE & $3.11 \mathrm{E}-11$ & $1.06 \mathrm{E}-17$ & $1.35 \mathrm{E}-14$ & 0 & 4.53 & 13.84 \\
\hline Methanol & $\mathrm{CH} 3 \mathrm{OH}$ & $9.02 \mathrm{E}-13$ & 0 & 0 & 0 & 22.23 & 11.79 \\
\hline Ethene & $\mathrm{C} 2 \mathrm{H} 4$ & $7.74 \mathrm{E}-12$ & $1.68 \mathrm{E}-18$ & $2.24 \mathrm{E}-16$ & 0 & 9.22 & 8.38 \\
\hline Acetaldehyde & $\mathrm{CH} 3 \mathrm{CHO}$ & $1.48 \mathrm{E}-11$ & 0 & $2.84 \mathrm{E}-15$ & $1.98 \mathrm{E}-05$ & 15.44 & 7.86 \\
\hline Acetic Acid & $\mathrm{CH} 3 \mathrm{CO} 2 \mathrm{H}$ & $8.00 \mathrm{E}-13$ & 0 & 0 & 0 & 7.64 & 6.03 \\
\hline Ethyne & $\mathrm{C} 2 \mathrm{H} 2$ & $7.46 \mathrm{E}-13$ & 0 & 0 & 0 & 4.97 & 5.96 \\
\hline Formic Acid & $\mathrm{HCOOH}$ & $4.50 \mathrm{E}-13$ & 0 & 0 & 0 & 3.89 & 4.86 \\
\hline Acrolein & $\mathrm{ACR}$ & $2.00 \mathrm{E}-11$ & $2.90 \mathrm{E}-19$ & $3.26 \mathrm{E}-15$ & $3.86 \mathrm{E}-08$ & 3.24 & 4.43 \\
\hline 1-propene & $\mathrm{C} 3 \mathrm{H} 6$ & $2.83 \mathrm{E}-11$ & $1.04 \mathrm{E}-17$ & $9.79 \mathrm{E}-15$ & 0 & 7.52 & 2.64 \\
\hline 2-furfural & FURFURAL & $3.50 \mathrm{E}-11$ & 0 & 0 & 1.34E-01 & 2.07 & 2.03 \\
\hline Acetone & $\mathrm{CH} 3 \mathrm{COCH} 3$ & $1.78 \mathrm{E}-13$ & 0 & 0 & 7.97E-05 & 5.60 & 1.95 \\
\hline Furan & FURAN & $4.20 \mathrm{E}-11$ & 0 & 0 & 0 & 2.26 & 1.87 \\
\hline Furanone & BZFUONE & $4.45 \mathrm{E}-11$ & $2.20 \mathrm{E}-19$ & $3.00 \mathrm{E}-13$ & 0 & 1.77 & 1.73 \\
\hline 2,3-butanedione & BIACET & $2.41 \mathrm{E}-13$ & 0 & 0 & $2.55 \mathrm{E}-05$ & 2.09 & 1.66 \\
\hline 1,3-butadiene & $\mathrm{C} 4 \mathrm{H} 6$ & $6.59 \mathrm{E}-11$ & $6.64 \mathrm{E}-18$ & $1.03 \mathrm{E}-13$ & 0 & 2.40 & 1.42 \\
\hline Ethanol & $\mathrm{C} 2 \mathrm{H} 5 \mathrm{OH}$ & $3.21 \mathrm{E}-12$ & 0 & 0 & 0 & 1.63 & 1.34 \\
\hline Glyoxal & GLYOX & $9.63 \mathrm{E}-12$ & 0 & $2.84 \mathrm{E}-15$ & $6.95 \mathrm{E}-05$ & 0.98 & 1.25 \\
\hline Hydroxyacetone & ACETOL & $4.42 \mathrm{E}-12$ & 0 & 0 & $4.55 \mathrm{E}-05$ & 1.30 & 1.12 \\
\hline Guaiacol & GUAIACOL & 7.44E-11 & 0 & 0 & 0 & 2.94 & 1.10 \\
\hline 5-methylfurfural & MEFURFURAL & $5.10 \mathrm{E}-11$ & 0 & 0 & 0 & 1.45 & 1.00 \\
\hline Catechol & CATECHOL & $1.00 \mathrm{E}-10$ & $9.20 \mathrm{E}-18$ & $9.90 \mathrm{E}-11$ & 0 & 1.45 & 1.00 \\
\hline Phenol & PHENOL & $2.74 \mathrm{E}-11$ & 0 & $3.80 \mathrm{E}-12$ & 0 & 2.39 & 0.89 \\
\hline Methyl acetate & METHACET & $3.50 \mathrm{E}-13$ & 0 & 0 & 0 & 1.03 & 0.89 \\
\hline Propenoic acid & $\mathrm{ACO} 2 \mathrm{H}$ & $8.66 \mathrm{E}-12$ & 0 & 0 & 0 & 0.87 & 0.86 \\
\hline Methyl vinyl ketone & MVK & $1.99 \mathrm{E}-11$ & $5.36 \mathrm{E}-18$ & 0 & $8.06 \mathrm{E}-06$ & 0.99 & 0.81 \\
\hline
\end{tabular}


Table S1. (Continued)

\begin{tabular}{|c|c|c|c|c|c|c|c|}
\hline & MCM Name & $\mathrm{k}_{\mathrm{OH}}\left(\mathrm{cm}^{3} \operatorname{molec}^{-1} \mathrm{~s}^{-1}\right)$ & $\mathrm{k}_{\mathrm{O} 3}\left(\mathrm{~cm}^{3}\right.$ molec $\left.^{-1} \mathrm{~s}^{-1}\right)$ & $\mathrm{k}_{\mathrm{NO} 3}\left(\mathrm{~cm}^{3} \mathrm{molec}^{-1} \mathrm{~s}^{-1}\right)$ & $\mathrm{j}\left(\mathrm{s}^{-1}\right)$ & $\mathrm{F} 26(\mathrm{ppb})$ & F38 (ppb) \\
\hline o-cresol & CRESOL & $4.65 \mathrm{E}-11$ & 0 & $1.40 \mathrm{E}-11$ & 0 & 2.58 & 0.79 \\
\hline alpha-pinene & APINENE & $5.20 \mathrm{E}-11$ & $9.53 \mathrm{E}-17$ & $6.15 \mathrm{E}-12$ & 0 & 0.76 & 0.78 \\
\hline Methyl glyoxal & MGLYOX & $1.29 \mathrm{E}-11$ & 0 & $6.82 \mathrm{E}-15$ & $1.20 \mathrm{E}-04$ & 0.69 & 0.69 \\
\hline Benzene & BENZENE & $1.22 \mathrm{E}-12$ & 0 & 0 & 0 & 1.93 & 0.67 \\
\hline 2-methylfuran & MEFURAN & $6.19 \mathrm{E}-11$ & 0 & 0 & 0 & 1.35 & 0.64 \\
\hline Toluene & TOLUENE & $5.59 \mathrm{E}-12$ & 0 & 0 & 0 & 1.90 & 0.46 \\
\hline Isoprene & $\mathrm{C} 5 \mathrm{H} 8$ & $9.91 \mathrm{E}-11$ & $1.33 \mathrm{E}-17$ & $7.03 \mathrm{E}-13$ & 0 & 1.04 & 0.42 \\
\hline Methyl ethyl ketone & MEK & $1.11 \mathrm{E}-12$ & 0 & 0 & $4.55 \mathrm{E}-05$ & 1.47 & 0.41 \\
\hline Acetic Anhydride & METHCOACET & $1.00 \mathrm{E}-14$ & 0 & 0 & 0 & 0.33 & 0.30 \\
\hline p-benzoquinone & PBZQONE & $4.60 \mathrm{E}-12$ & 0 & $3.00 \mathrm{E}-13$ & 0 & 0.24 & 0.30 \\
\hline 2,5-dimethylfuran & DIMEFURAN & $1.32 \mathrm{E}-10$ & 0 & 0 & 0 & 0.75 & 0.28 \\
\hline Methacrolein & MACR & $2.84 \mathrm{E}-11$ & $1.28 \mathrm{E}-18$ & $3.40 \mathrm{E}-15$ & $7.72 \mathrm{E}-08$ & 0.29 & 0.24 \\
\hline Benzaldehyde & BENZAL & $1.25 \mathrm{E}-11$ & 0 & $2.40 \mathrm{E}-15$ & $3.58 \mathrm{E}-04$ & 0.28 & 0.19 \\
\hline 2,3-dimethyl phenol & OXYLOL & $8.00 \mathrm{E}-11$ & 0 & $3.20 \mathrm{E}-11$ & 0 & 0.71 & 0.16 \\
\hline Propenal & C4ALDB & $3.40 \mathrm{E}-11$ & $1.58 \mathrm{E}-18$ & $6.00 \mathrm{E}-15$ & $5.56 \mathrm{E}-08$ & 0.18 & 0.15 \\
\hline Styrene & STYRENE & $5.80 \mathrm{E}-11$ & $1.70 \mathrm{E}-17$ & $1.50 \mathrm{E}-12$ & 0 & 0.36 & 0.11 \\
\hline 1-pentene & PENT1ENE & $3.10 \mathrm{E}-11$ & $1.00 \mathrm{E}-17$ & $1.20 \mathrm{E}-14$ & 0 & 0.34 & 0.10 \\
\hline m-xylene & MXYL & $2.31 \mathrm{E}-11$ & 0 & $2.60 \mathrm{E}-16$ & 0 & 0.26 & 0.09 \\
\hline p-xylene & PXYL & $1.43 \mathrm{E}-11$ & 0 & $5.00 \mathrm{E}-16$ & 0 & 0.26 & 0.09 \\
\hline o-xylene & OXYL & $1.36 \mathrm{E}-11$ & 0 & $4.10 \mathrm{E}-16$ & 0 & 0.17 & 0.06 \\
\hline Ethyl benzene & EBENZ & $7.00 \mathrm{E}-12$ & 0 & $1.20 \mathrm{E}-16$ & 0 & 0.08 & 0.03 \\
\hline NO2 & $\mathrm{NO} 2$ & $9.22 \mathrm{E}-12$ & $3.72 \mathrm{E}-17$ & $1.21 \mathrm{E}-12$ & $5.05 \mathrm{E}-05$ & 6.46 & 53.98 \\
\hline $\mathbf{O 3}$ & $\mathrm{O} 3$ & $7.41 \mathrm{E}-14$ & $4.96 \mathrm{E}-02$ & 0 & 0 & 5.00 & 10.00 \\
\hline HONO & HONO & $5.95 \mathrm{E}-12$ & 0 & 0 & $5.90 \mathrm{E}-04$ & 0.00 & 2.58 \\
\hline NO & NO & $8.97 \mathrm{E}-12$ & $1.78 \mathrm{E}-14$ & $2.60 \mathrm{E}-11$ & 0 & 0.08 & 0.40 \\
\hline
\end{tabular}


Table S2. Calculated NMOG losses by reaction with $\mathrm{OH}, \mathrm{O}_{3}, \mathrm{NO}_{3}$, and photolysis for F26, F38, and the ambient biomass burning plume described by Müller et al. (2016). All values are percentages of the integrated loss over $15 \mathrm{hr}$ of atmospheric-equivalent $\mathrm{OH}$ oxidation. Entries are ordered according to the largest loss rates by each process, calculated for F38. Entries marked by a hyphen were not included in the modeling.

Primary Loss by $\mathrm{OH}$

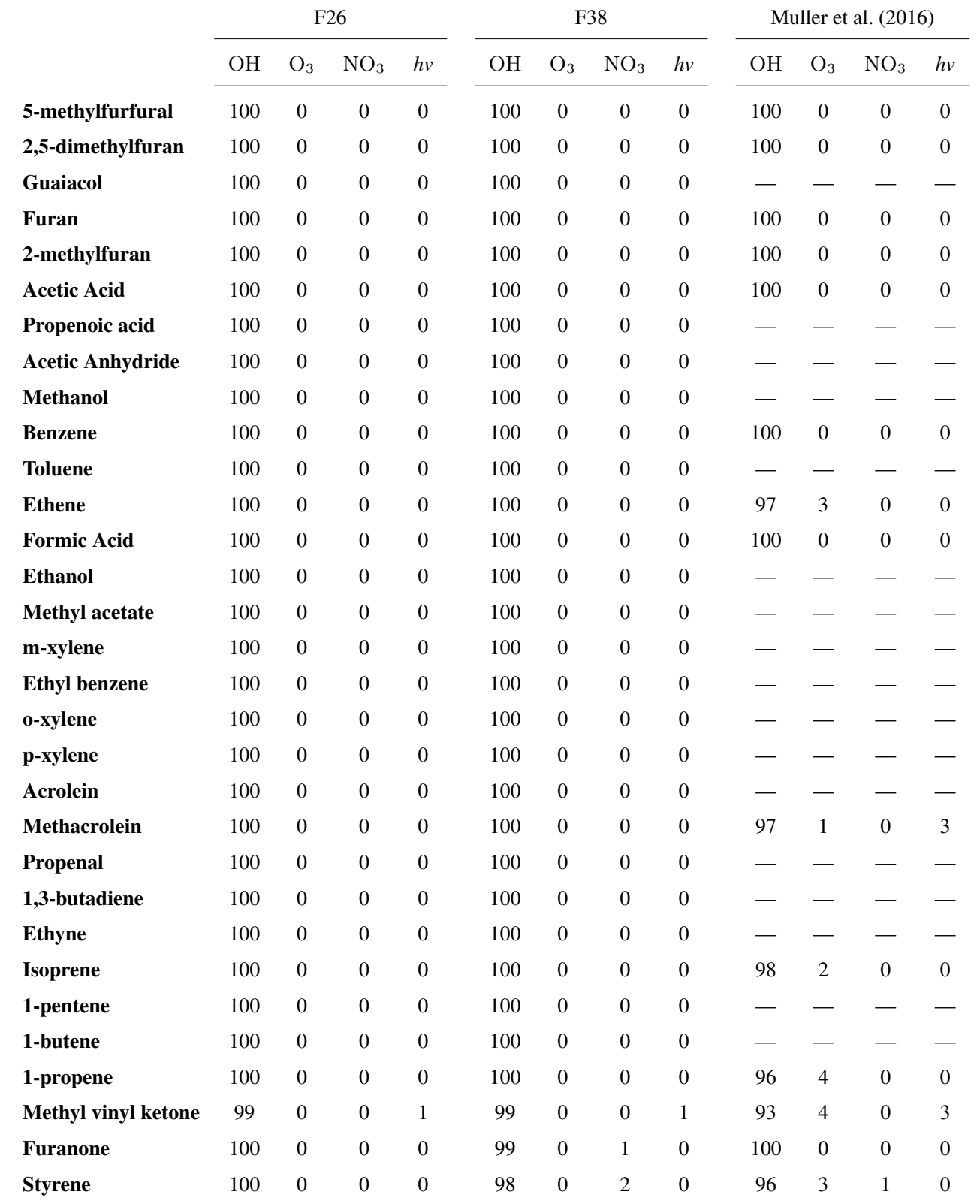


Table S2. (Continued)

Significant loss by NO3

Catechol
o-cresol
2,3-dimethyl phenol
p-benzoquinone
Phenol
alpha-pinene

\begin{tabular}{cccc}
\multicolumn{4}{c}{$\mathrm{F} 26$} \\
\hline $\mathrm{OH}$ & $\mathrm{O}_{3}$ & $\mathrm{NO}_{3}$ & $h v$ \\
\hline 93 & 0 & 7 & 0 \\
98 & 0 & 2 & 0 \\
98 & 0 & 2 & 0 \\
99 & 0 & 1 & 0 \\
99 & 0 & 1 & 0 \\
98 & 1 & 1 & 0
\end{tabular}

\begin{tabular}{cccc}
\multicolumn{4}{c}{$\mathrm{F} 38$} \\
\hline $\mathrm{OH}$ & $\mathrm{O}_{3}$ & $\mathrm{NO}_{3}$ & $h v$ \\
\hline 66 & 0 & 34 & 0 \\
83 & 0 & 17 & 0 \\
84 & 0 & 16 & 0 \\
91 & 0 & 9 & 0 \\
88 & 0 & 12 & 0 \\
92 & 1 & 7 & 0
\end{tabular}

\begin{tabular}{cccc}
\multicolumn{5}{c}{ Muller et al. (2016) } \\
\hline $\mathrm{OH}$ & $\mathrm{O}_{3}$ & $\mathrm{NO}_{3}$ & $h v$ \\
\hline 82 & 1 & 17 & 0 \\
94 & 0 & 6 & 0 \\
- & - & - & - \\
- & - & - & - \\
97 & 0 & 3 & 0 \\
- & - & - & -
\end{tabular}

Significant loss by photolysis

\begin{tabular}{lcccc}
\cline { 2 - 5 } 2-furfural & 0 & 0 & 0 & 100 \\
Acetone & 18 & 0 & 0 & 82 \\
2,3-butanedione & 48 & 0 & 0 & 52 \\
Methyl ethyl ketone & 70 & 0 & 0 & 30 \\
Benzaldehyde & 74 & 0 & 0 & 26 \\
Hydroxyacetone & 93 & 0 & 0 & 7 \\
Methyl glyoxal & 93 & 0 & 0 & 7 \\
Glyoxal & 95 & 0 & 0 & 5 \\
Formaldehyde & 99 & 0 & 0 & 1 \\
Acetaldehyde & 98 & 0 & 0 & 2
\end{tabular}

\begin{tabular}{cccc}
\multicolumn{4}{c}{$\mathrm{F} 38$} \\
\hline $\mathrm{OH}$ & $\mathrm{O}_{3}$ & $\mathrm{NO}_{3}$ & $h v$ \\
\hline 1 & 0 & 0 & 99 \\
16 & 0 & 0 & 84 \\
46 & 0 & 0 & 54 \\
68 & 0 & 0 & 32 \\
73 & 0 & 0 & 27 \\
91 & 0 & 0 & 9 \\
91 & 0 & 0 & 9 \\
93 & 0 & 0 & 7 \\
98 & 0 & 0 & 2 \\
98 & 0 & 0 & 2
\end{tabular}

\begin{tabular}{cccc}
\multicolumn{5}{c}{ Muller et al. (2016) } \\
\hline $\mathrm{OH}$ & $\mathrm{O}_{3}$ & $\mathrm{NO}_{3}$ & $h v$ \\
\hline 99 & 0 & 0 & 1 \\
77 & 0 & 0 & 23 \\
1 & 0 & 0 & 99 \\
- & - & - & - \\
- & - & - & - \\
88 & 0 & 0 & 12 \\
- & - & - & - \\
- & - & - & - \\
39 & 0 & 0 & 61 \\
95 & 0 & 0 & 5
\end{tabular}




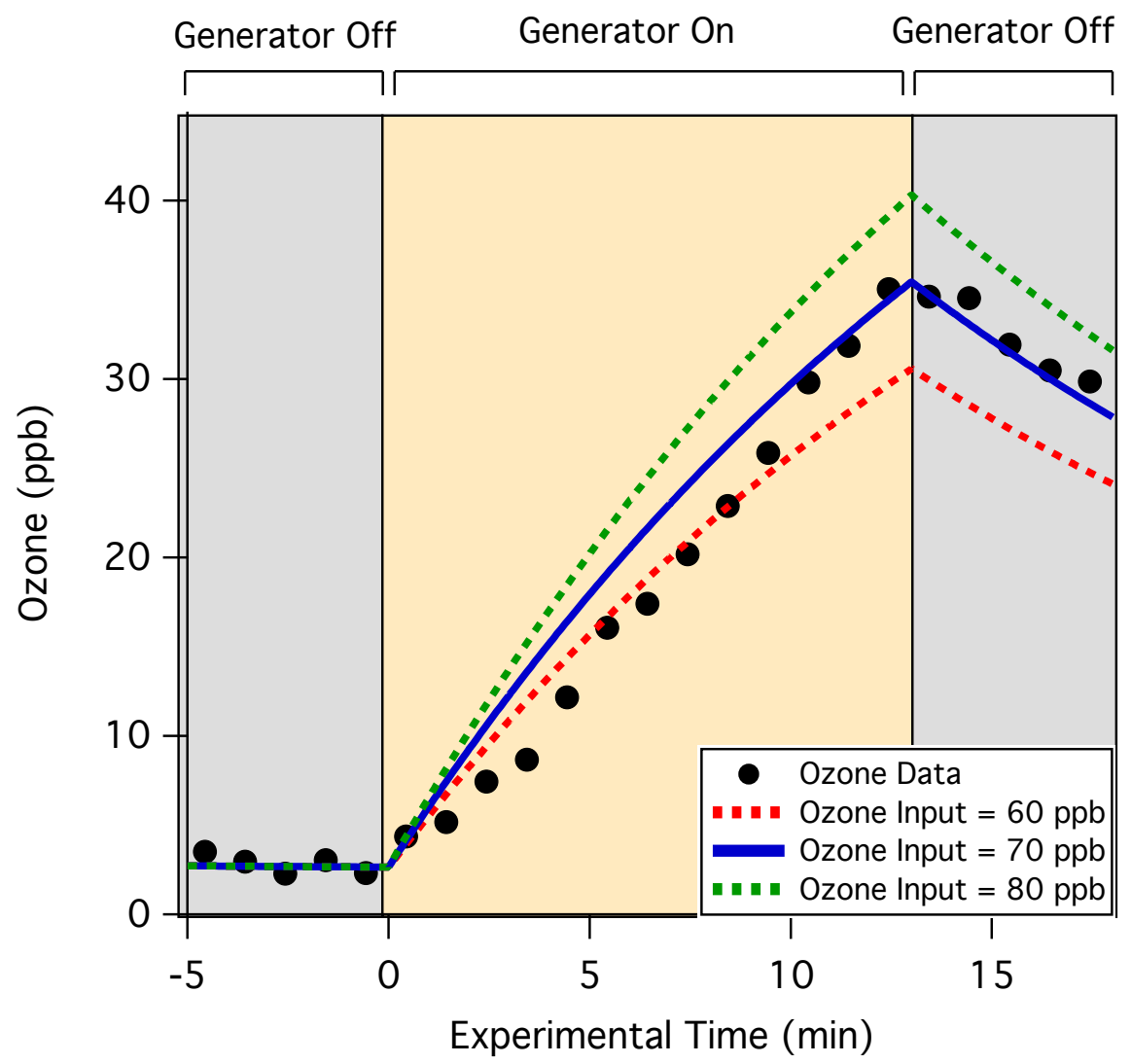

Figure S1. Modeled ozone compared to ozone measured during a dark, low NMOG $(<70 \mathrm{ppb})$ experiment. Output from the model is shown assuming that the dilution stream contains 60,70 , and $80 \mathrm{ppb}$ of ozone. The input of ozone with the best fit (70 ppb) is applied to the photochemistry model described in Section 2.4 


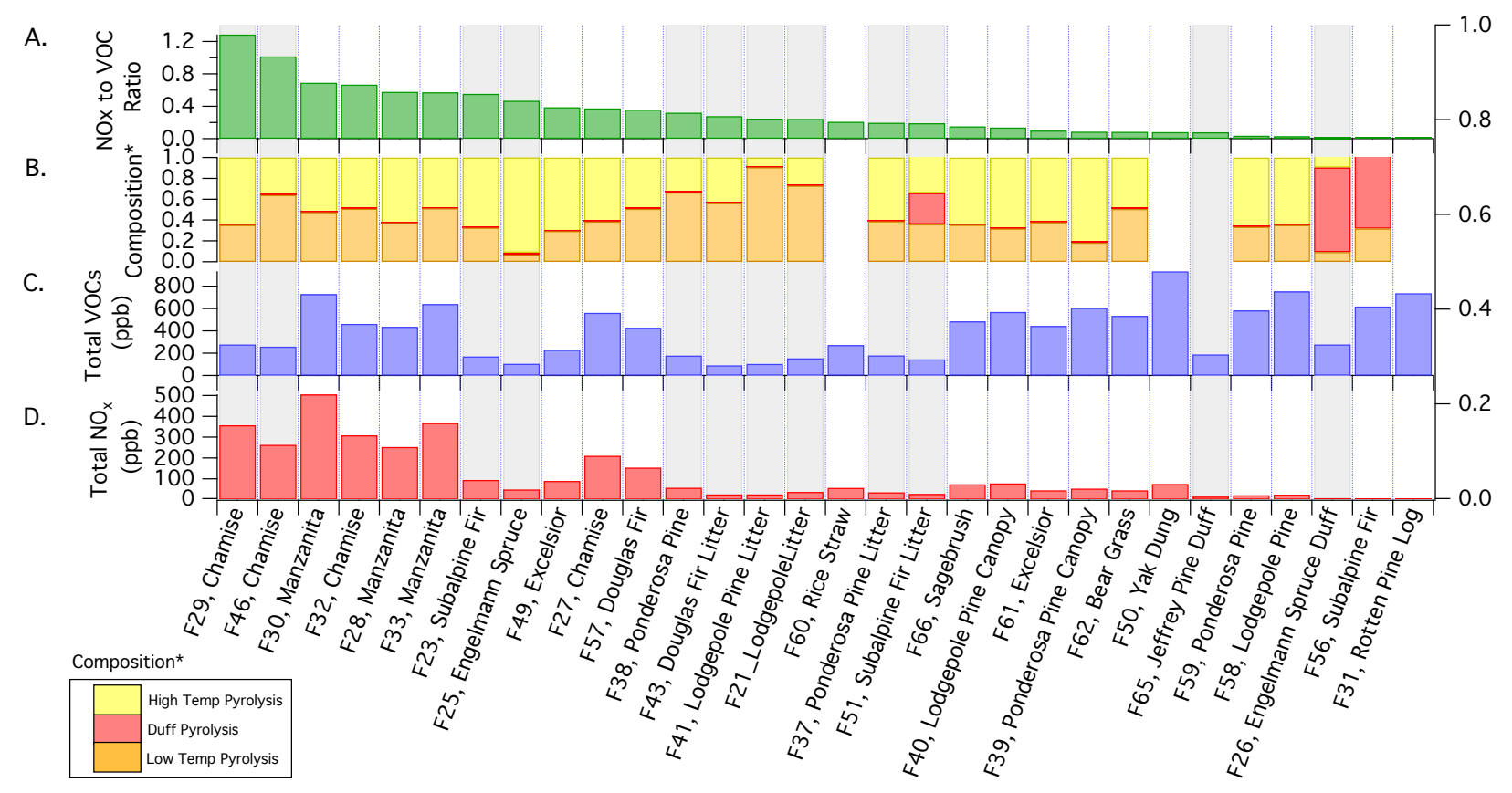

Figure S2. Small chamber (A) $\mathrm{NO}_{\mathrm{x}} / \mathrm{NMOG}$ ratio, (B) NMOG composition, (C) total NMOG loading, and (D) total $\mathrm{NO}_{\mathrm{x}}$ prior to photochemical oxidation. Panel B shows the fraction of the total NMOG signal attributable to high temperature, low temperature, and duff pyrolysis as defined by Sekimoto et al. (2017). The grey bars indicate experiments in which initial NMOG loadings are sufficiently low to avoid significant $\mathrm{OH}$ titration. 


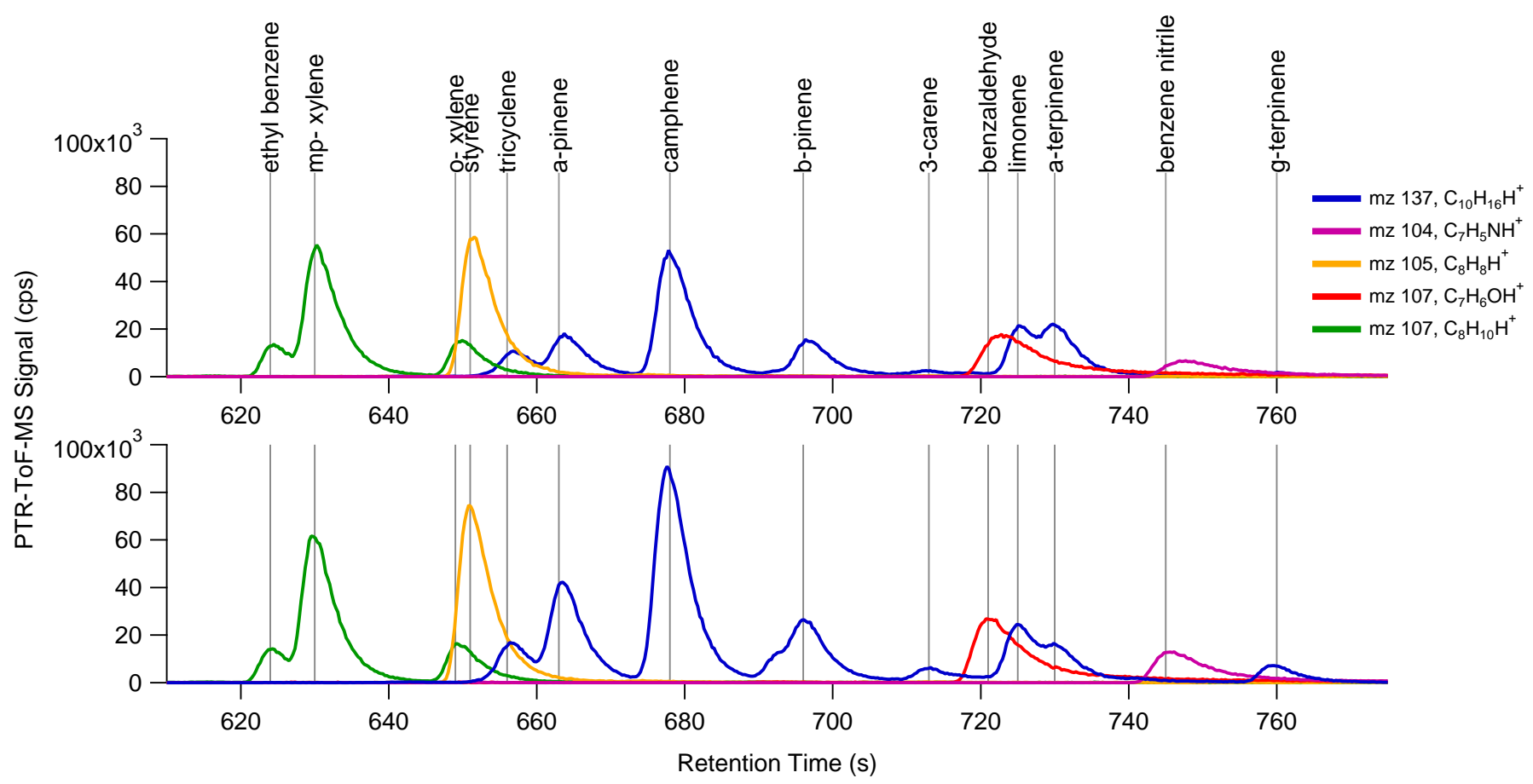

Figure S3. Distirbution of monoterpenes $(\mathrm{m} / \mathrm{z}, 137)$ and other select NMOG measured from the combustion of douglas (top) and subalpine fir (bottom) using GC-PTR-ToF-MS. 


\section{Furan Reactions}
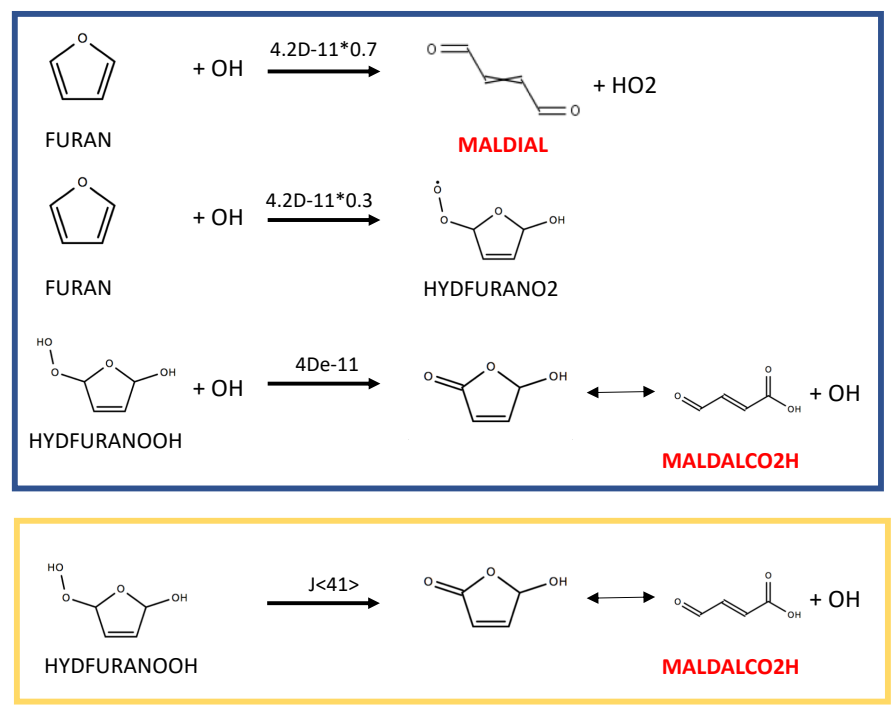

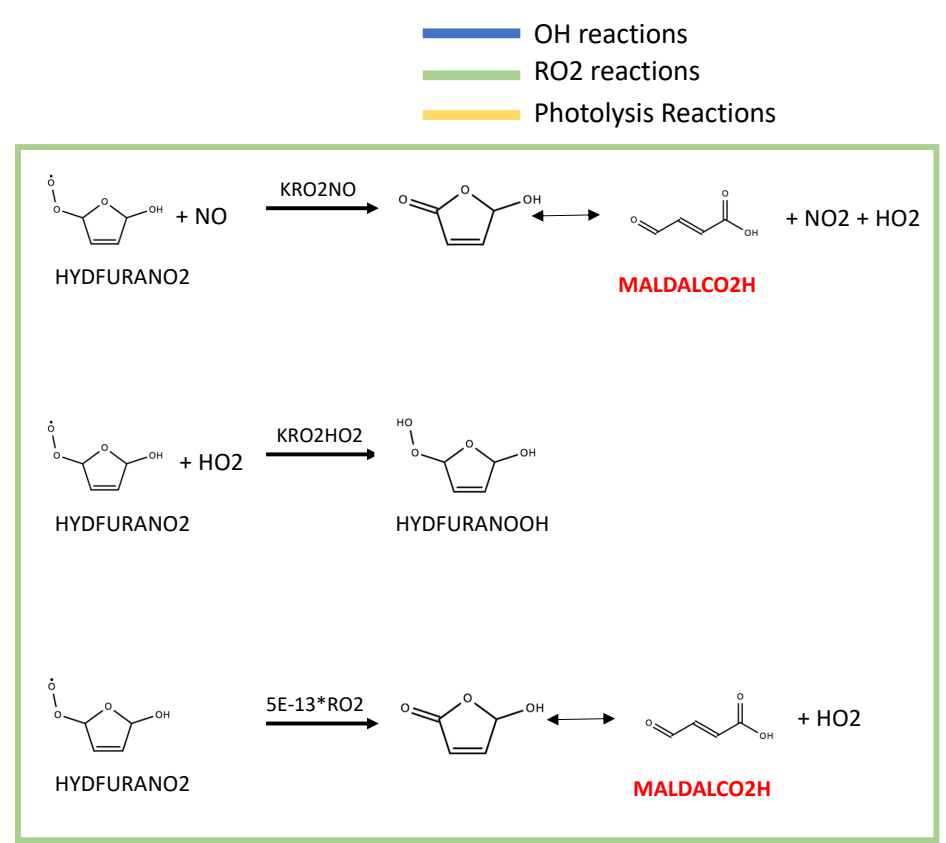

Figure S4. Furan reactions implemented into the MCM box model. Reactions are based on mechanism reported by Aschmann et al. (2014). Names in red indicate species currently represented in MCM v 3.3.1. 


\section{Methyl Furan Reactions}

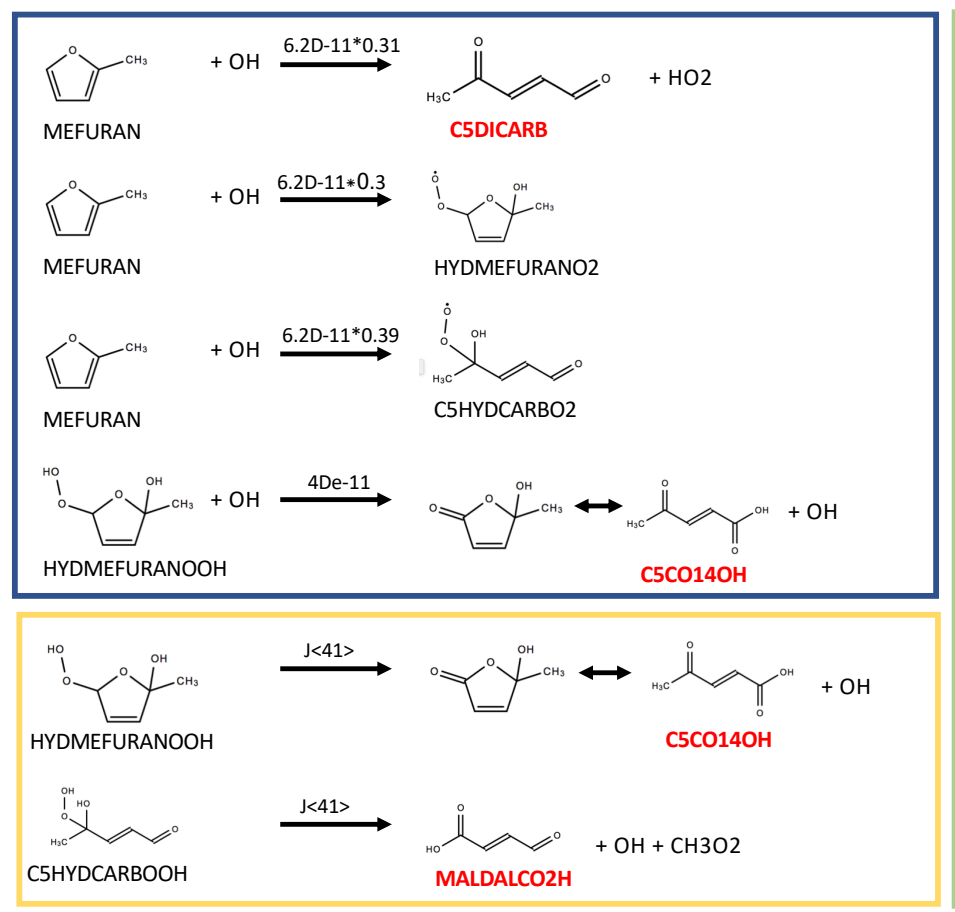

$\mathrm{OH}$ reactions $\mathrm{RO} 2$ reactions Photolysis Reactions

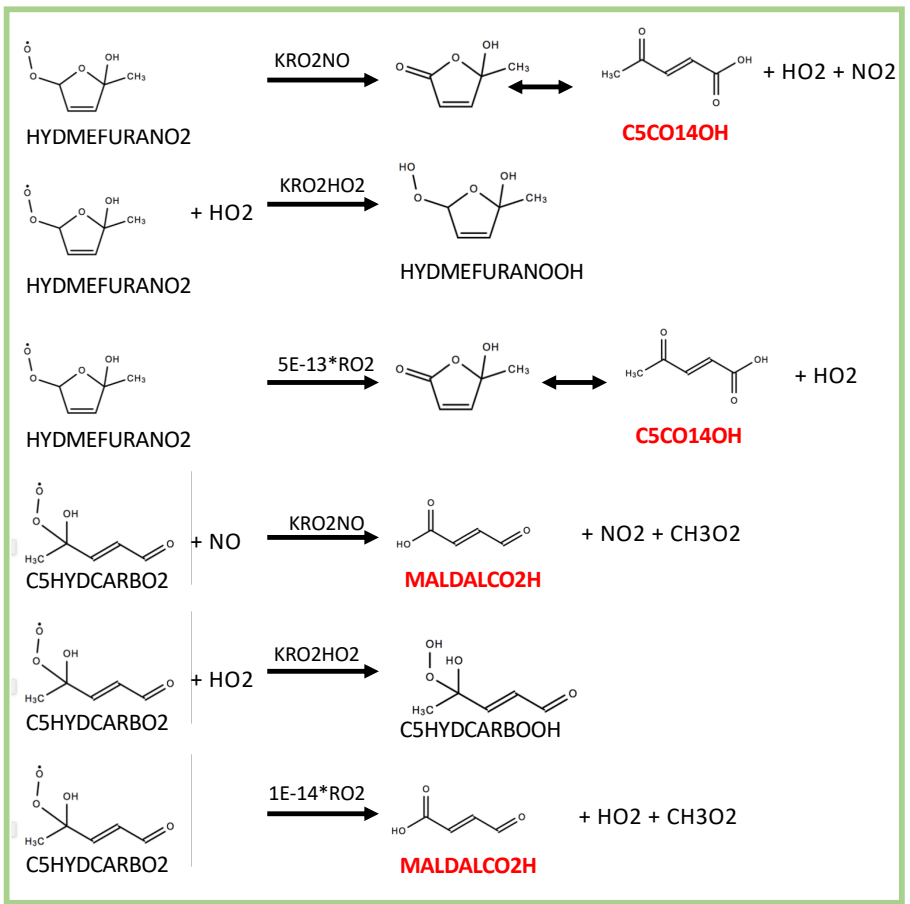

Figure S5. 2-methylfuran reactions implemented into the MCM box model. Reactions are based on mechanism reported by Aschmann et al. (2014). Names in red indicate species currently represented in MCM v 3.3.1. 


\section{Dimethyl Furan Reactions}
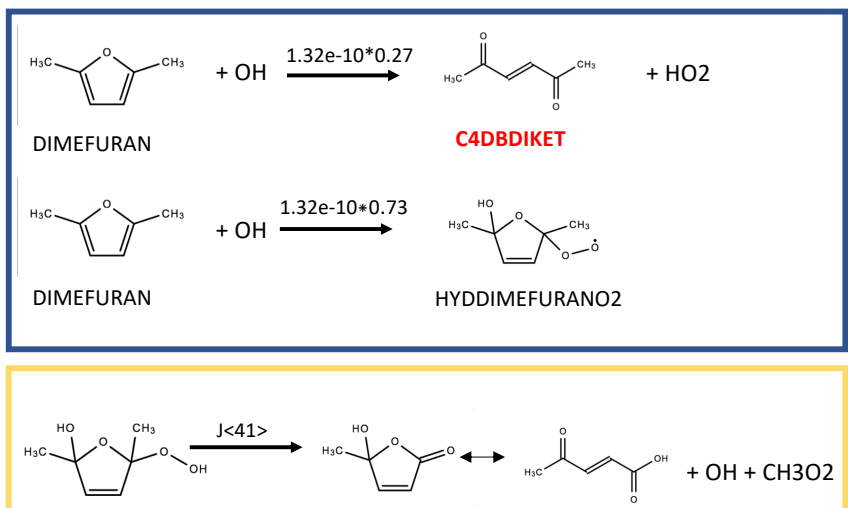

HYDDIMEFURANOOH

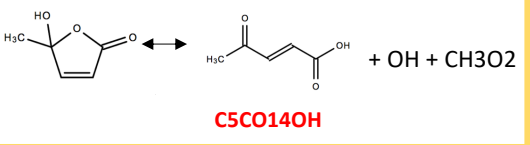

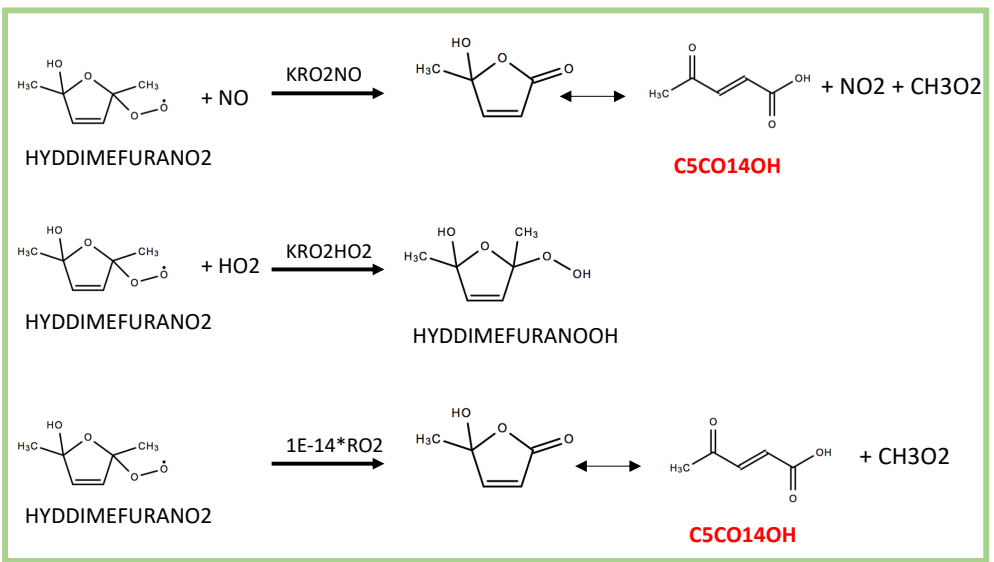

Figure S6. 2,5-dimethylfuran reactions implemented into the MCM box model. Reactions are based on mechanism reported by Aschmann et al. (2014). Names in red indicate species currently represented in MCM v 3.3.1. 


\section{Furfural Reactions}
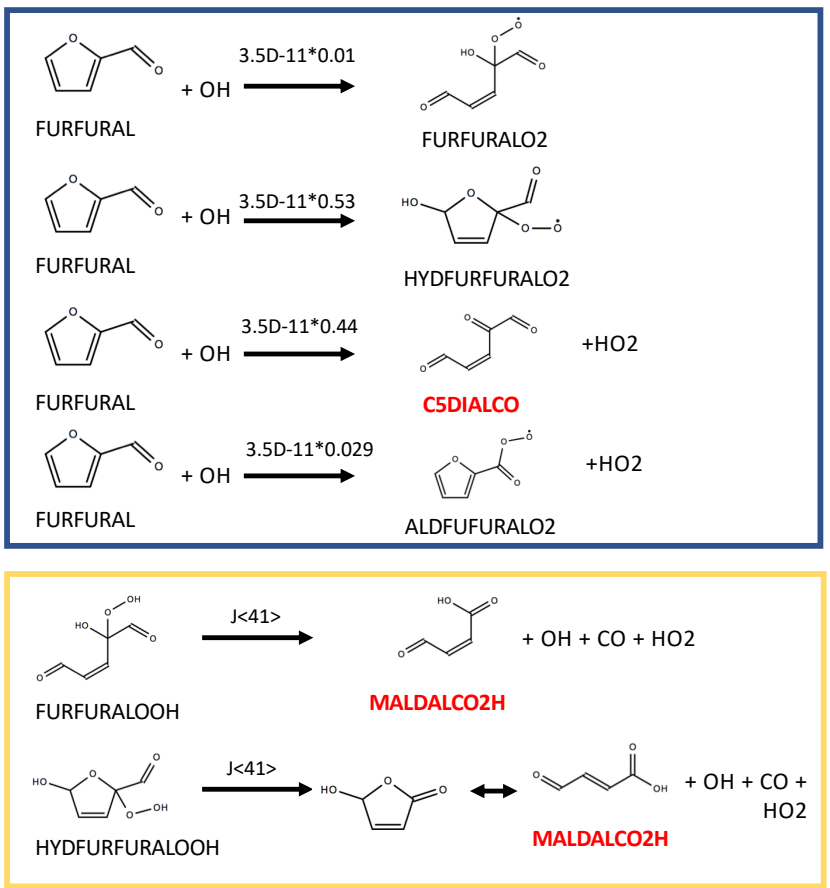

$\mathrm{OH}$ reactions

$\mathrm{RO} 2$ reactions

Photolysis Reactions

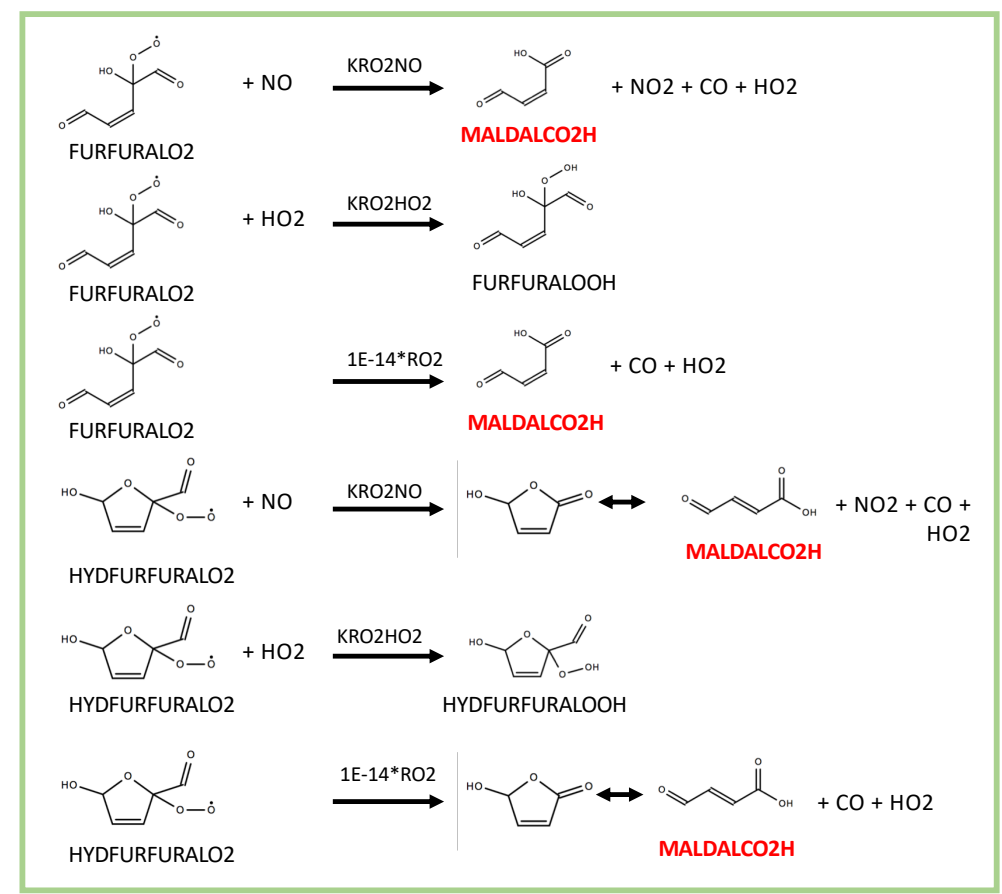

Figure S7. Furfural reactions implemented into the MCM box model. Reactions are based on mechanism reported by Zhao and Wang (2017). Names in red indicate species currently represented in MCM v 3.3.1. 


\section{Furfural Reactions (Continued)}

$\mathrm{OH}$ reactions

$\mathrm{RO} 2$ reactions

Photolysis Reactions

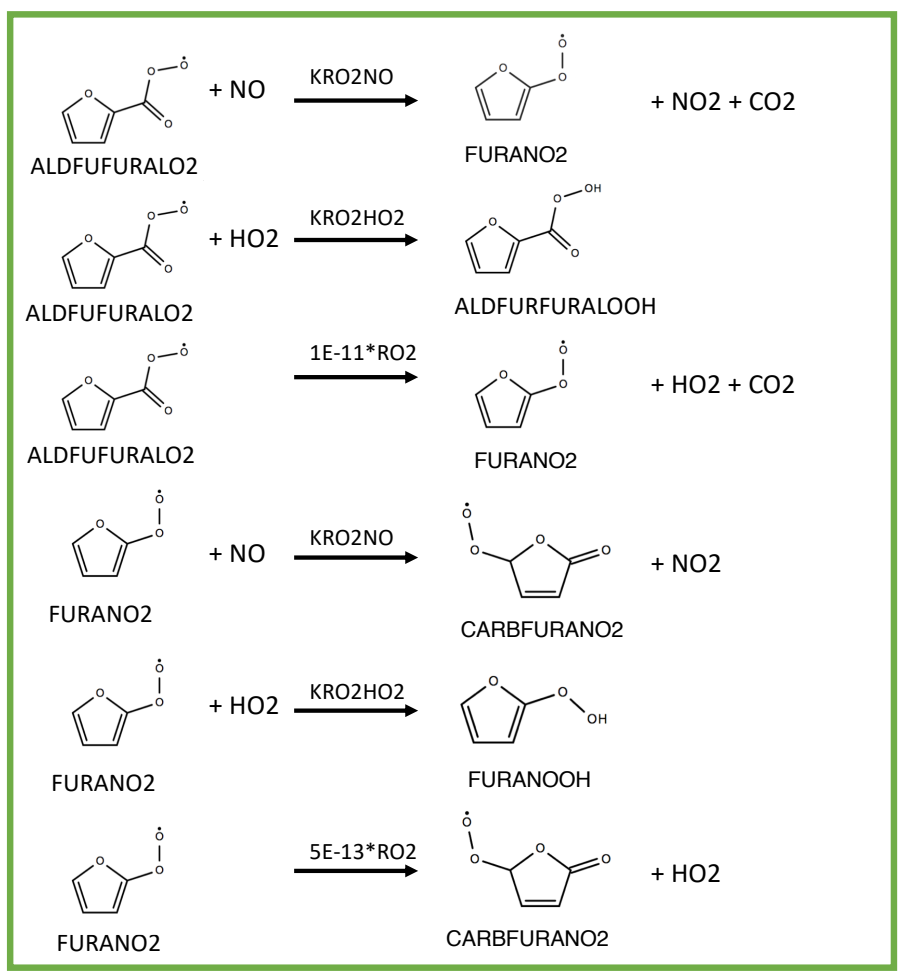

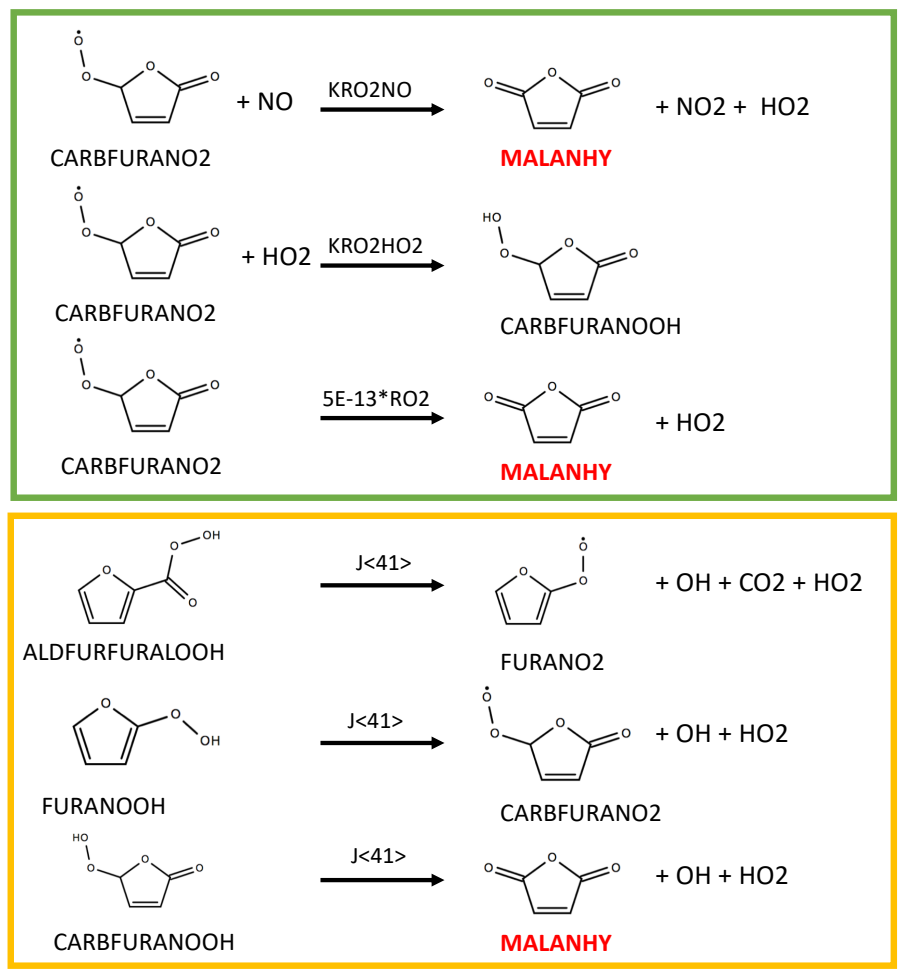

Figure S8. Furfural reactions implemented into the MCM box model (continued from Fig. S7s). Reactions are based on mechanism reported by Zhao and Wang (2017). Names in red indicate species currently represented in MCM v 3.3.1. 


\section{Methyl Furfural Reactions}
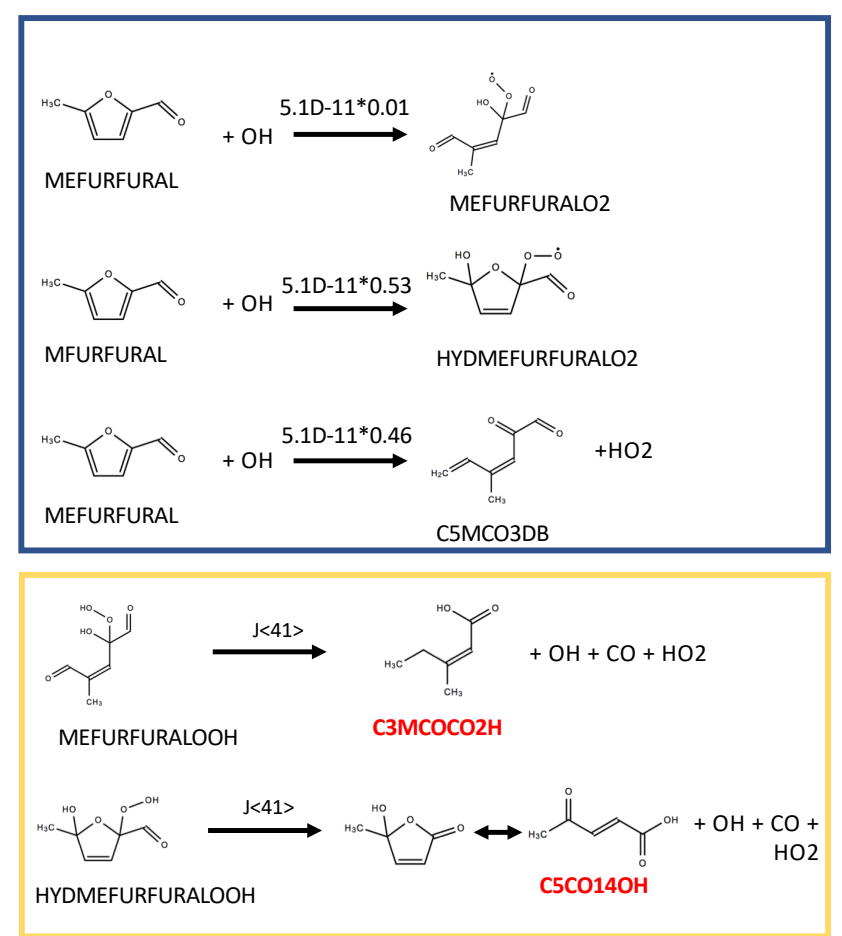

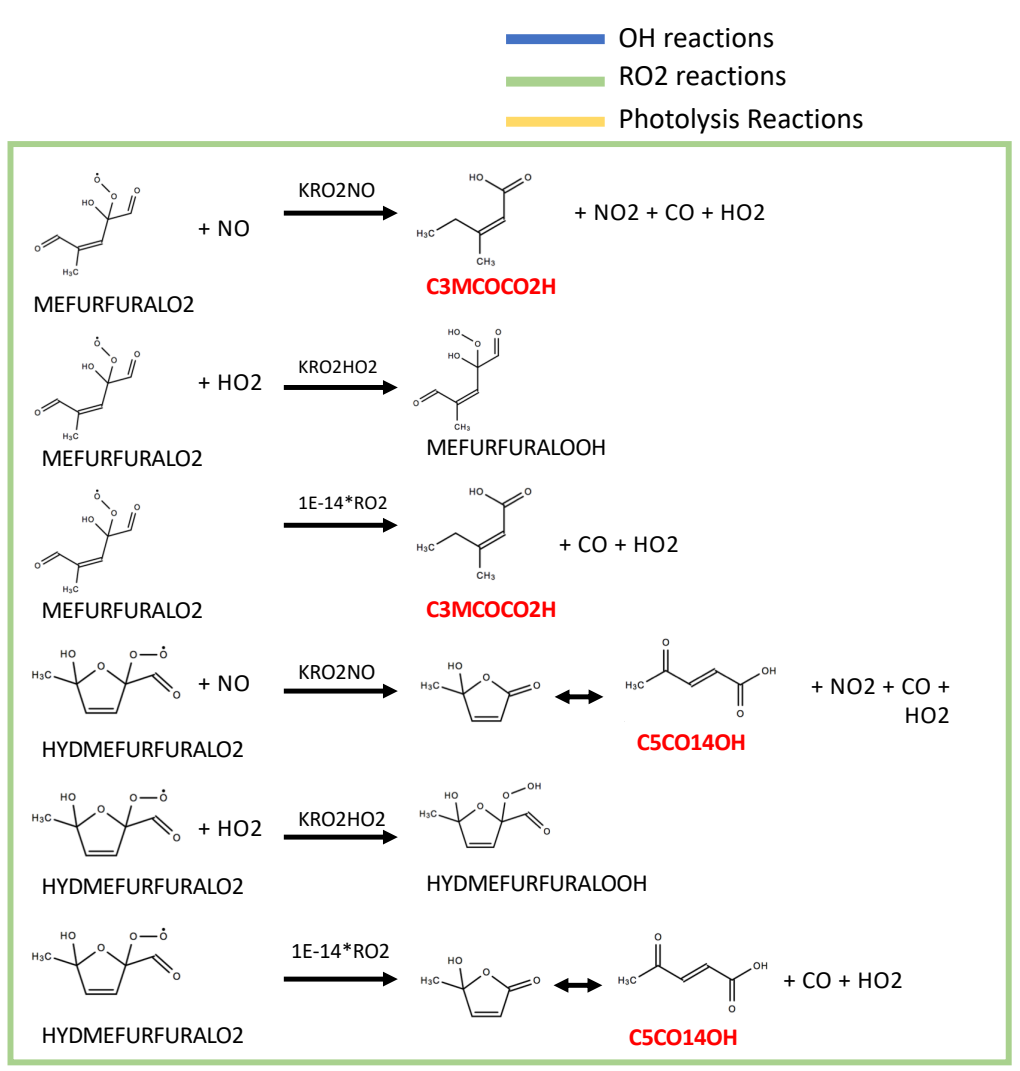

Figure S9. 5-methylfurfural reactions implemented into the MCM box model. Products and branching ratios are assumed to follow pathways analogous to the furfural mechanism reported by Zhao and Wang (2017). Names in red indicate species currently represented in MCM v 3.3.1. 


\section{Guaiacol Reactions $\quad$ RO2 reactions}
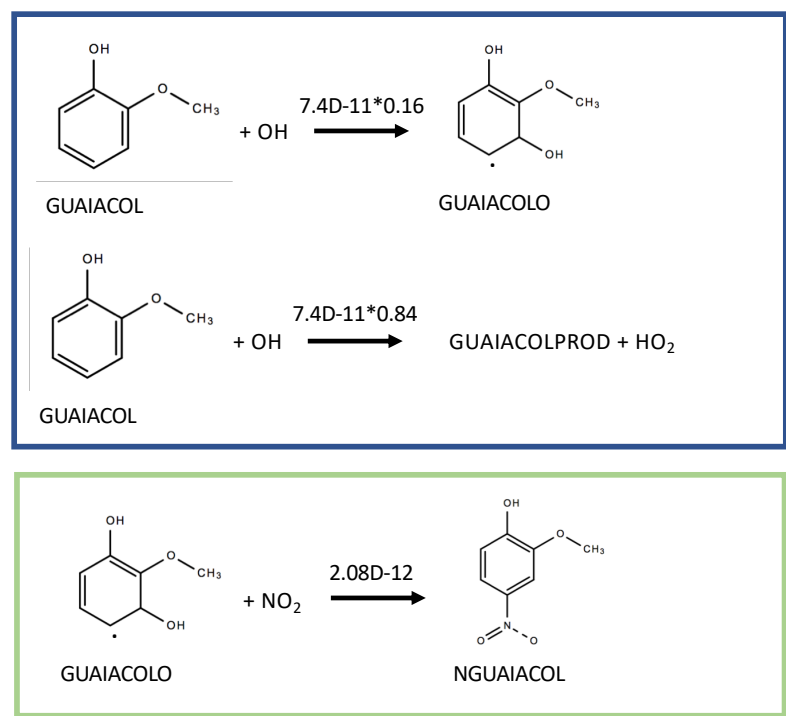

Figure S10. Guaiacol reactions implemented into the MCM box model. Reactions are based on the guaiacol mechanism reported by Lauraguais et al. (2014). 


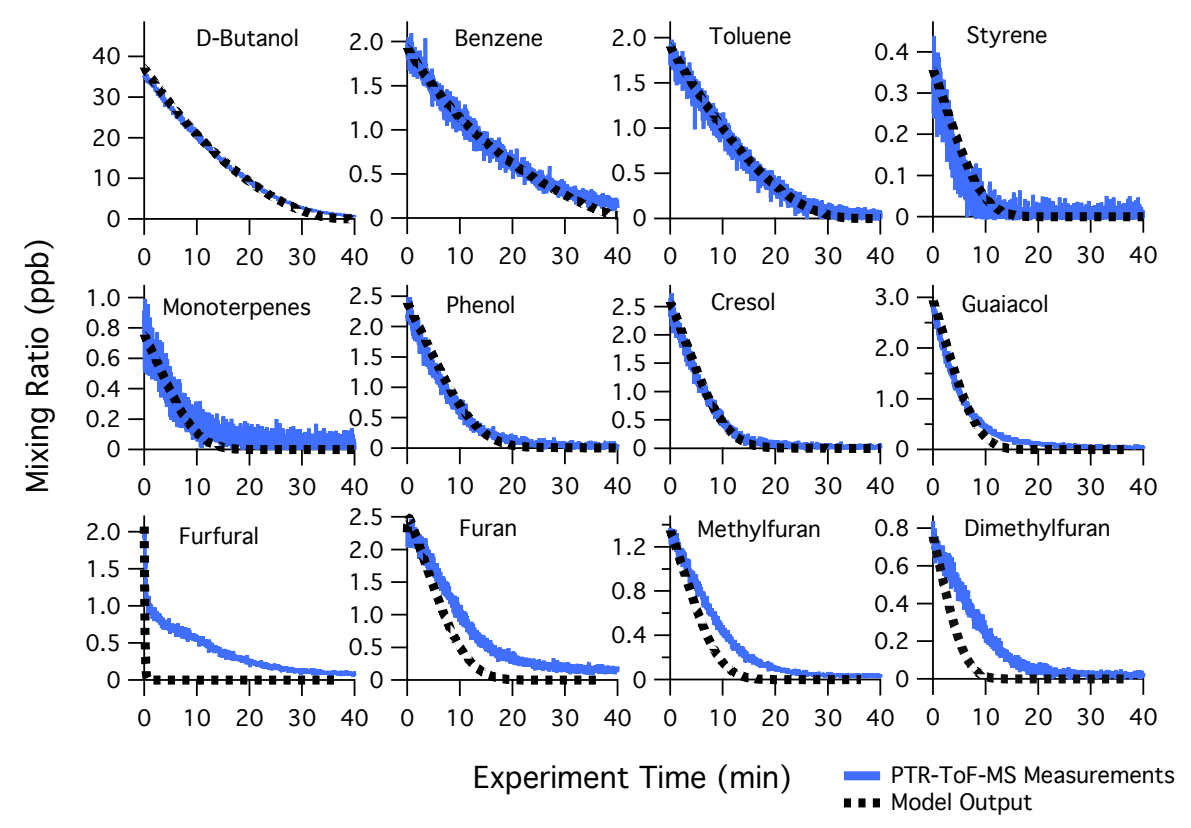

Figure S11. Primary NMOG measurements (blue lines) compared to modeled output (black dotted lines) for Fire 26. Fuel = Englemann Spruce Duff, $\mathrm{NO}_{\mathrm{x}} / \mathrm{NMOG}=0.02$, primarily duff pyrolysis $\mathrm{NMOG}$ mixture. 

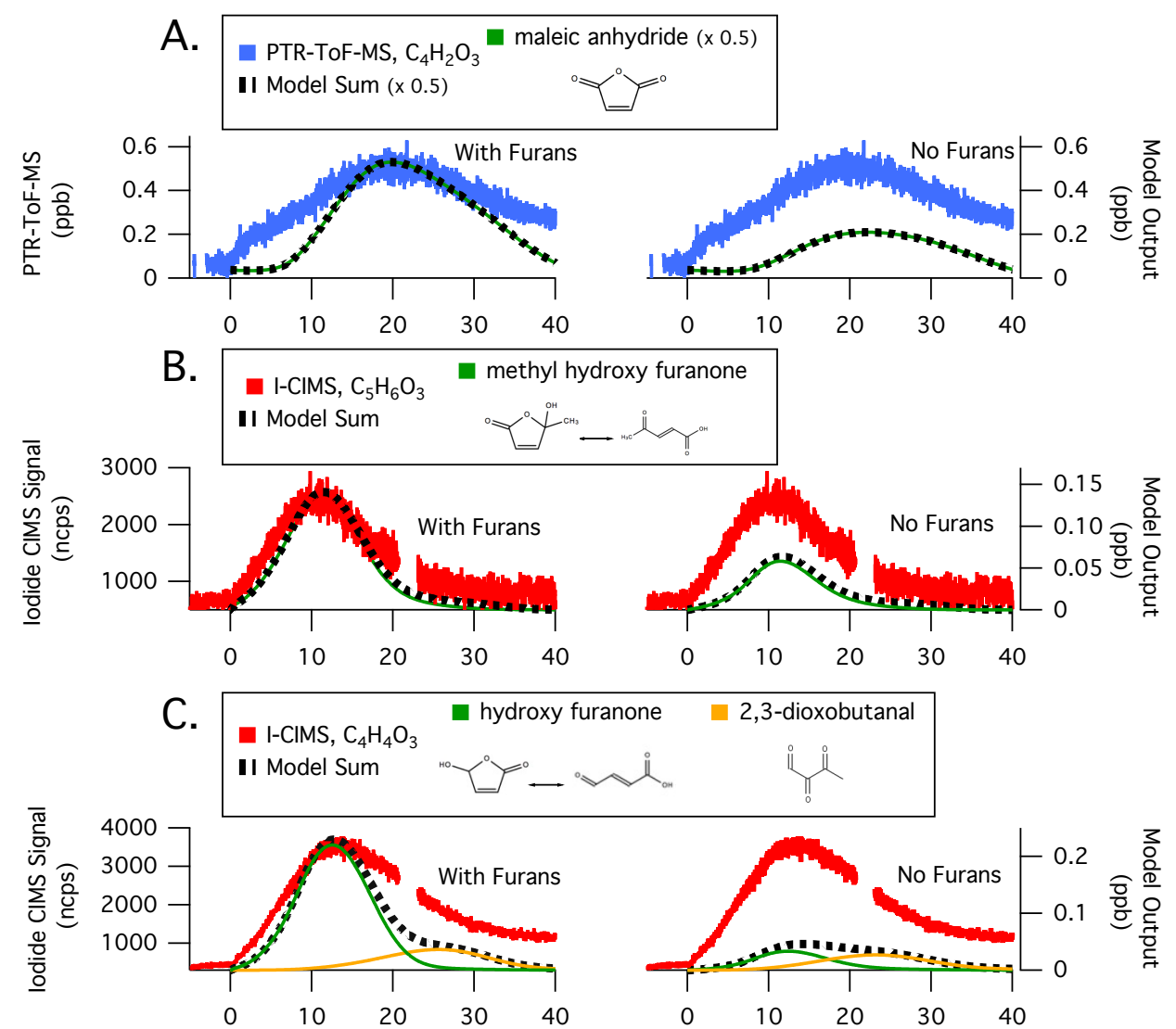

Experimental Time (min)

Figure S12. Secondary NMOG measurements compared to modeled output for Fire 26. Row (A) shows PTR-ToF-MS measurements of $\mathrm{C}_{4} \mathrm{H}_{2} \mathrm{O}_{3}$ compared to model output of maleic anhydride. Row (B) shows I-ToF-CIMS measurements of $\mathrm{C}_{5} \mathrm{H}_{6} \mathrm{O}_{3}$ compared to model output of methyl hydroxy furanone and its tautomer, $\beta$-acetylacrylic acid. Row (C) shows I-ToF-CIMS measurements of $\mathrm{C}_{4} \mathrm{H}_{4} \mathrm{O}_{3}$ compared to model output of hydroxy furanone, its tautomer malealdehydic acid, and 2,3-dioxobutanal. All graphs to the left show full model runs, while graphs to the right show model runs when the initial conditions of furan, 2-methylfuran, 2,5-dimethylfuran, furfural, 5-methylfurfural, and furanone are set to zero 

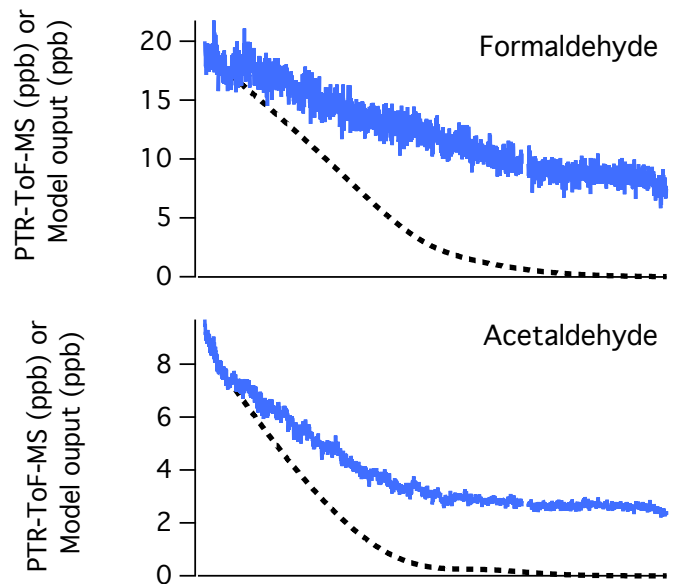

Acetaldehyde
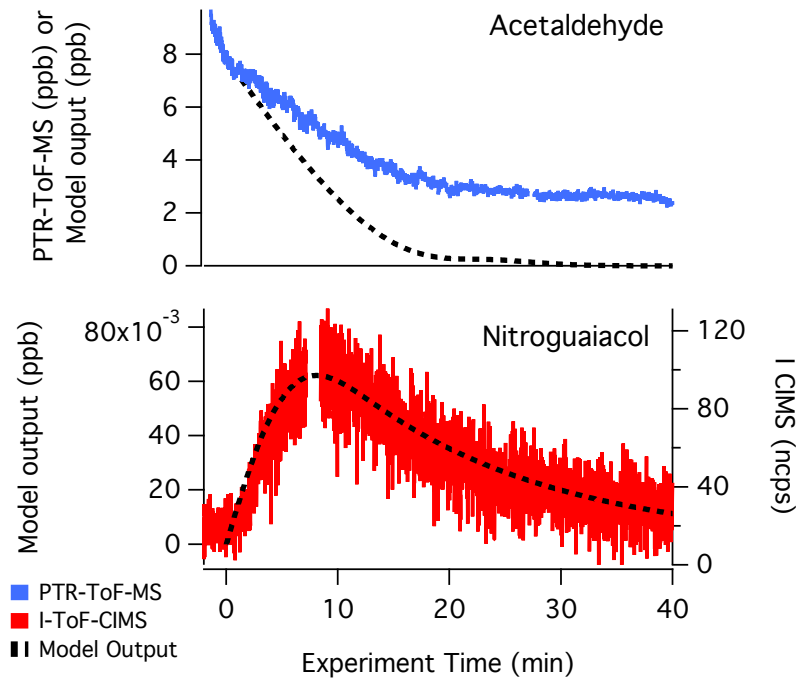

Figure S13. PTR-ToF-MS and $\mathrm{I}^{-}$-ToF-CIMS measurements of formaldehyde, acetaldehyde, and nitroguaiacol compared to model ouput for F38. 


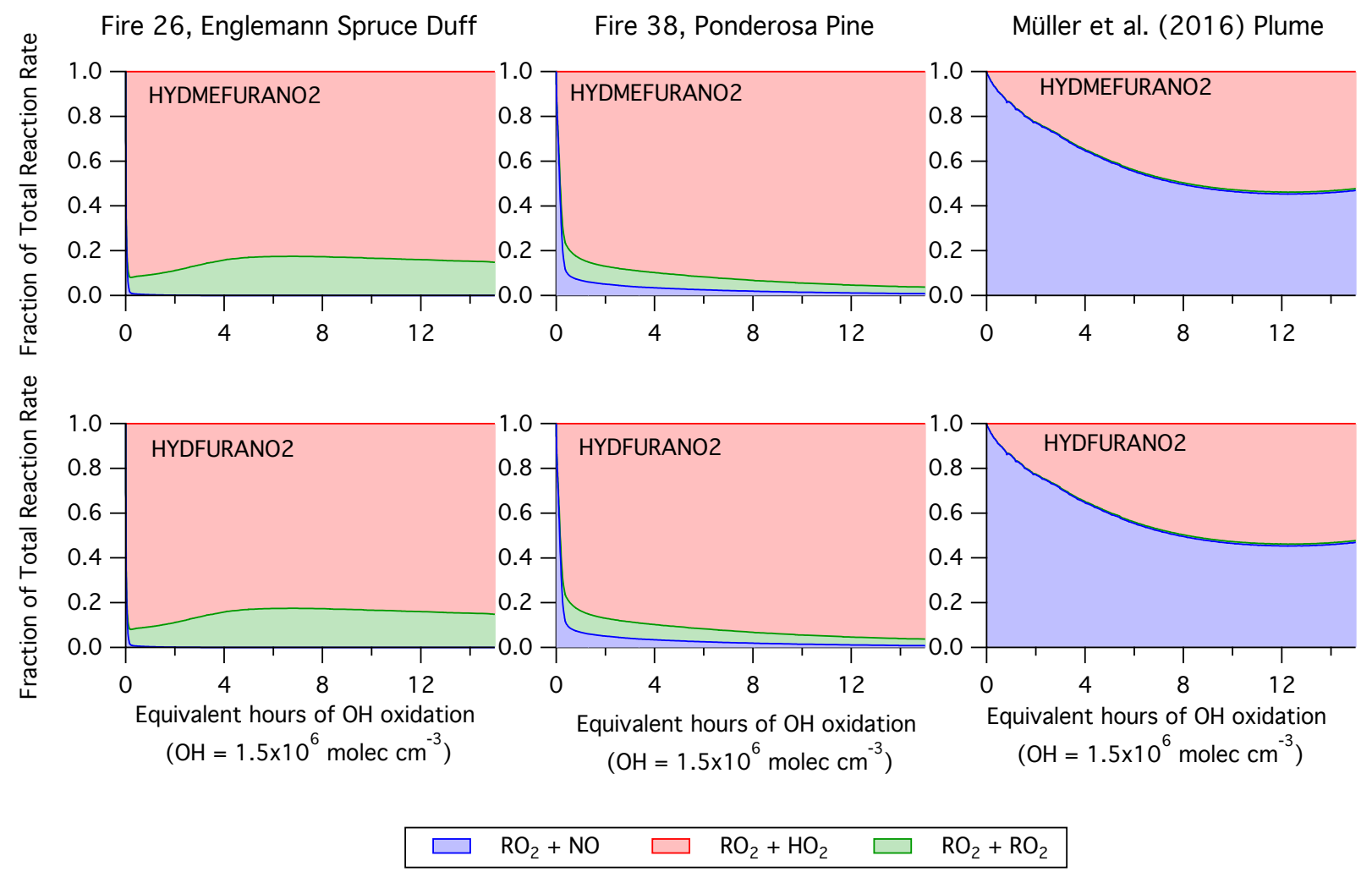

Figure S14. Fate of $\mathrm{RO}_{2}$ species that lead to the formation of hydroxy furanone and methyl hydroxy furanone for F26, F38, and the ambient plume described by Müller et al. (2016). Shown is the fraction of $\mathrm{RO}_{2}$ loss associated with reactions with $\mathrm{HO}_{2}, \mathrm{NO}$, and other $\mathrm{RO}_{2}$ radicals. 
A. F66 Sagebrush
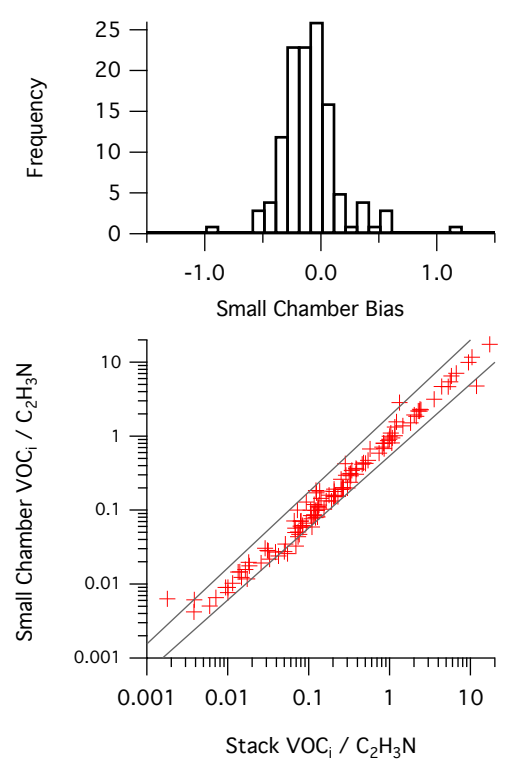

B. F26 Englman Spruce Duff
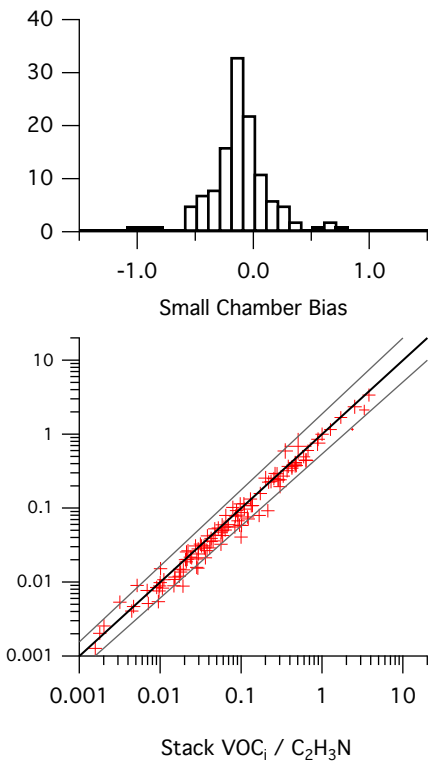

C. F29 Chamise
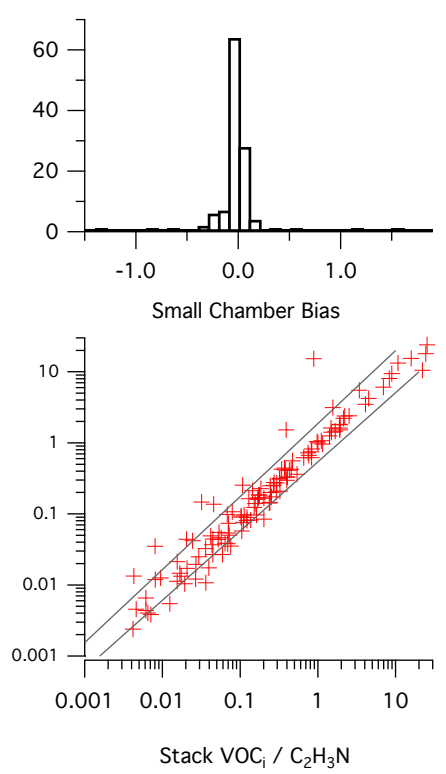

Figure S15. Comparison of NMOG distributions measured in the stack and small chamber prior to OH oxidation for (A) F66 - Sagebrush, (B) F26 - Englemann Spruce Duff, and (C) F29 Chamise. The bottom row shows the NMOG/ $\mathrm{C}_{2} \mathrm{H}_{3} \mathrm{~N}$ ratio for each species measured by the PTR-ToF-MS, along with 1:1, 2:1, and 1:2 lines. 

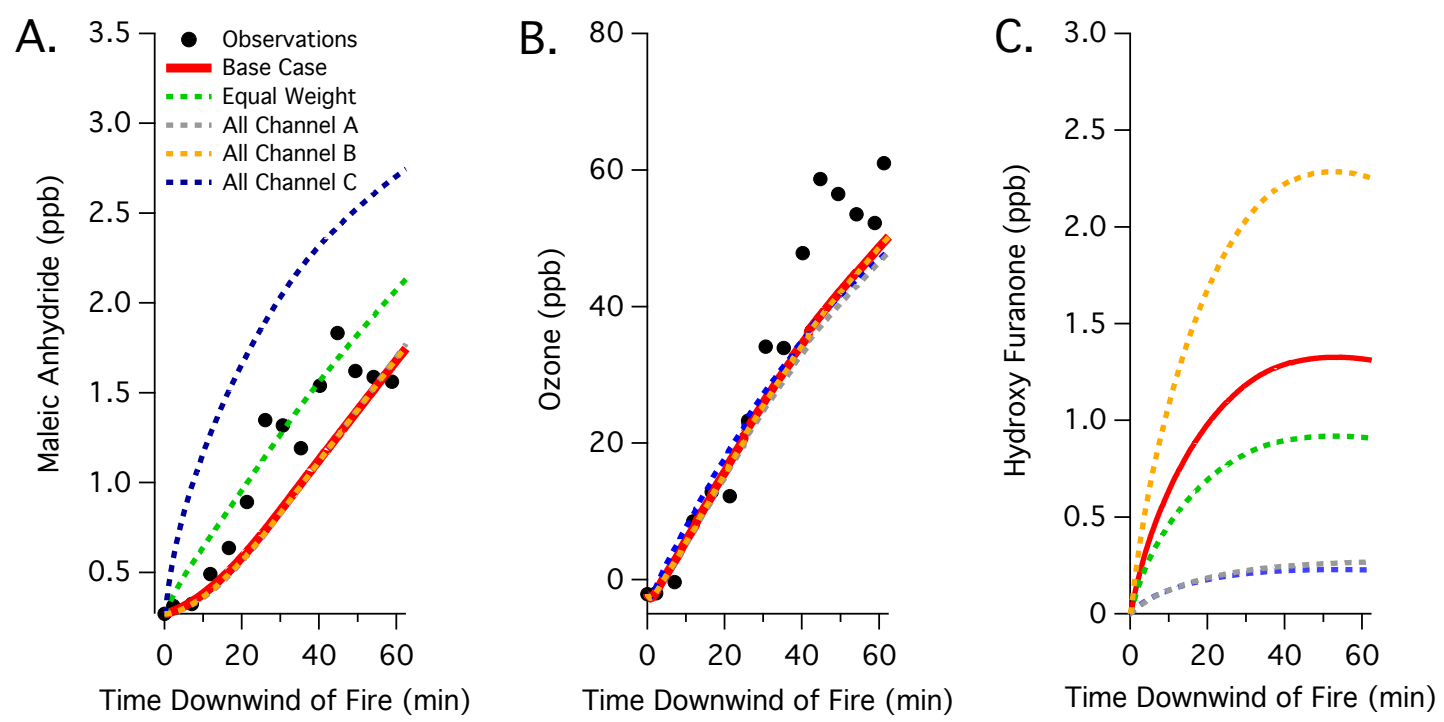

Figure S16. Model sensitivity of (A) maleic anhydride, (B) ozone, and (C) hydroxyfuranone to the assumed branching ratios of the furfural mechanism (Fig. 6). The "base case" simulation assumes branching ratios of $\mathrm{A}=0.37, \mathrm{~B}=0.6, \mathrm{~B}=0.03$ while the "equal weight" simulation assumes $\mathrm{A}=0.33, \mathrm{~B}=0.33, \mathrm{C}=0.33$. All other simulations assume that furfural loss follows a single channel (i.e., all channel $\mathrm{A}$, $\mathrm{B}$, or $\mathrm{C}$ ). Model output are compared to the measurements of maleic anhydride and ozone reported by Müller et al. (2016). Measurements of hydroxy furanone are not available. 